\title{
Automated Generation of Optimal Controllers through Model Checking Techniques
}

\author{
Giuseppe Della Penna ${ }^{1}$, Daniele Magazzeni ${ }^{1}$, Alberto Tofani ${ }^{1}$, Benedetto Intrigila ${ }^{2}$ \\ Igor Melatti ${ }^{3}$ and Enrico Tronci ${ }^{3}$ \\ ${ }^{1}$ Dipartimento di Informatica, Università di L'Aquila, Italy \\ \{dellapenna, magazzeni, tofani\}@di.univaq.it \\ ${ }^{2}$ Dipartimento di Matematica Pura ed Applicata, Università di Roma “Tor Vergata”, Italy \\ intrigilemat.uniroma2.it \\ ${ }^{3}$ Dipartimento di Informatica, Università di Roma "La Sapienza", Italy \\ \{melatti, tronci\}adi.uniroma1.it
}

\begin{abstract}
We present a methodology for the synthesis of controllers, which exploits (explicit) model checking techniques. That is, we can cope with the systematic exploration of a very large state space. This methodology can be applied to systems where other approaches fail. In particular, we can consider systems with an highly non-linear dynamics and lacking a uniform mathematical description (model). We can also consider situations where the required control action cannot be specified as a local action, and rather a kind of planning is required. Our methodology individuates first a raw optimal controller, then extends it to obtain a more robust one. A case study is presented which considers the well known truck-trailer obstacle avoidance parking problem, in a parking lot with obstacles on it. The complex non-linear dynamics of the truck-trailer system, within the presence of obstacles, makes the parking problem extremely hard. We show how, by our methodology, we can obtain optimal controllers with different degrees of robustness.
\end{abstract}

Keywords. Controller synthesis, controller optimization, model checking, nonlinear systems.

\section{Introduction}

Control systems (or, shortly, controllers) are small hardware/software components that control the behavior of larger systems, the plants. A controller continuously analyzes the plant state (looking at its state variables) and possibly adjusts some of its parameters (called control variables) to keep the system in a condition called setpoint, which usually represents the normal or correct behavior of the system.

In the last years, the use of sophisticated controllers has become very common in robotics, critical systems and, in general, in the hardware/software embedded systems contained in a growing number of everyday products and appliances.

However, since the primary aim of a controller is to ensure the correct behavior of the controlled plant, we have to guarantee the efficiency and the robustness of controllers. By efficiency, we mean the capability of the controller to bring the system to 
the set point in the shortest possible time (also called time optimality). By robustness, we mean the capability of the controller to perform well when the state variables vary outside the design range.

Therefore, the verification of these properties is a crucial task that is being addressed using different formal methodologies (e.g., model checking and theorem proving) developed in different research communities (e.g., automata theory and artificial intelligence [1]). In particular, much work is being done to provide a methodology for the automatic (or semi-automatic) synthesis of correct controllers directly from the plant specifications.

However, efficiency and robustness can hardly be simultaneously fulfilled, especially in critical systems, where the system dynamics is difficult to understand and to control.

Therefore a possible approach for approximate a correct solution, can be to start by synthesizing a very efficient or even optimal controller and - as a second step - to make it robust. (Observe, however, that in case safety is involved, a better approach could be to choose the best controller in the class of the safe ones [2]).

To this aim, in this chapter we describe an automatic methodology composed by:

1. a procedure for the synthesis of an optimal controller;

2. a procedure for the transformation of the optimal controller into a robust one.

Our methodology exploits explicit model checking in an innovative way w.r.t the approaches adopted so far, especially in the AI planning area. In particular, the first procedure - looking for an optimal solution - actually can also be considered as a planner. Indeed, this procedure does not simply individuate a good local move, but searches for the best possible sequence of actions to bring the plant to the setpoint. Therefore, it can be also used as a planner, though we will not pursue this point in this chapter.

\section{Controller Synthesis Techniques}

In this section we recall some basic notions about controller synthesis and describe the main results presented in this field by the recent research.

\subsection{Controller Synthesis}

There are a number of well-established techniques for the synthesis of controller. For short, we mention only three of them:

1. PID controllers;

2. fuzzy controllers;

3. dynamic programming techniques.

As it well-known [3], PID-based techniques are very effective for linear systems, while they badly perform w.r.t. non-linear ones. On the contrary our technique is able to cope with such systems, as shown in the case study.

Fuzzy control is well known as a powerful technique for designing and realizing control systems, especially suitable when a mathematical model is lacking or is too 
complex to allow an analytical treatment [4] [5]. However fuzzy rules correspond to local actions, so that, in general, they do not result in an optimal controller. Moreover there are situations where local actions are not viable at all, and rather a kind of planning is required. For an example of such a situation see the case study.

Dynamic programming techniques are very suitable for the generation of optimal controllers [6] [7]. Although our methodology has a dynamic programming flavor, it can cope with (and it is especially suitable for) very rough plant descriptions, whose mathematical definition cannot be adapted to the dynamic programming preconditions, when the cost function cannot be decomposed or the system dynamics function cannot be inverted. Again, see the case study, where a backward decomposition of the cost function (in this case, the length of the path) is hard to perform, due to the complexity of the system dynamics function and to the presence of obstacles. Indeed, we performed a direct systematic analysis of the trajectories, using model checking techniques to support the required computational effort.

\section{Optimal Controller Generation through Model Checking}

Our objective is to build an optimal controller for a system (or plant) $\mathcal{S}$ which, at every state, has a limited number of allowed actions. Moreover, we suppose that $\mathcal{S}$ starts at a given interesting initial state $s_{0}$, and that the final goal is to bring $\mathcal{S}$ in a goal state (however, we can easily generalize to the case with $n$ initial states). We recall that our optimality criterion is essentially the time optimality: that is, we want to bring $\mathcal{S}$ in a goal state in the smallest possible number of steps.

Thus, our controller has to be able to decide, for every state of $\mathcal{S}$ which is reachable from $s_{0}$, which is the action that brings to the nearest (w.r.t. the number of steps) goal state. The optimality of the action chosen implies the optimality of the generated controller. Note that forcing the controller to consider all the states reachable from $s_{0}$, instead of controlling only the states in the unique optimal path from $s_{0}$ to a goal state, allows us to handle the cases in which a bad move is made as a consequence of a given action.

In order to build such a controller, we consider the transition graph $G$ of $\mathcal{S}$, where the nodes are the reachable states and a transition between two nodes models an allowed action between the corresponding states. In this setting, the problem of designing the optimal controller reduces to finding the minimum path in $G$ between each state and the nearest goal state.

Unfortunately a transition graph for complex, real-world systems could be often huge, due to the well-known state explosion problem. Thus it is likely that $G$ would not fit into the available RAM memory, and then the minimum path finding process could be highly time-consuming.

However, Model Checking techniques [8] [9] [10] developed in the last decades have shown to be able to deal with very huge state spaces. Thus, our idea is to reuse such model checking algorithms, reshaping them to be a controller generator. Note that in this chapter we focus on protocol-based hybrid systems, so we use model checking techniques based on an on-the-fly explicit enumeration of the system under analysis, since for such kind of systems these algorithms often outperform the symbolic ones [11]. 
More in detail, in our technique we have two phases, which we describe in the following.

\subsection{Optimal Raw Controller Synthesis Phase}

In the first phase, an explicit model checking algorithm is used, which performs a Depth First (DF) visit of all the reachable states of $\mathcal{S}$, starting from $s_{0}$. As usual, a hash table $\mathrm{HT}$ is used in order to store already visited states. Moreover, the stack holds, together with states, also the next action to be explored.

However, the DF visit is enriched in order to generate the controller C. To this aim, HT also stores, for each visited state, a flag toGoal, initially set to 0 . When a goal state $g$ is reached, then the states in the current path from $s_{0}$ to $g$ (that is to say, the states currently on the stack) will have this flag set to 1 , as soon as the visit backtracks to them. This is to signify that such states indeed reach a goal, and may be put in $\mathrm{C}$ - together with the action taken and the number of steps they need to reach the goal itself. In this way, when a state $s$ with the toGoal flag set to 1 is reached, then we can analogously set the toGoal flag on all the states currently on the stack, and put them on $\mathrm{C}$.

However, this scheme may fail in the following case. Suppose that a cycle $s_{1} r_{1} \ldots$ $r_{h} s_{2} t_{1} \ldots t_{k} s_{1}$ is found, were $s_{1}$ and $s_{2}$ are on the stack. When analyzing $t_{k}$, the toGoal flag of $s_{1}$ is not set to 1 , since we have not backtracked from $s_{2}$ yet. However, the visit is truncated, since $s_{1}$ is already visited, thus $t_{k}$ will not be inserted in C (unless it was already present, or it reaches a goal through another path which does not intersect the stack).

To avoid this, a predecessor table PT is maintained for each state which is visited again while it is on the stack. We have that PT stores all the paths leading from a state on the stack and another state previously on the stack. Thus, in the situation described above, the path $r_{1} \ldots r_{h} s_{2} t_{1} \ldots t_{k}$ is added to the predecessor table of $s_{1}$. Thus, when the DF visit of $s_{1}$ is finished, all the states in its predecessor table are added on $\mathrm{C}$ by using a backward visit, provided that $s_{1}$ indeed reaches a goal.

Finally, in order to preserve optimality, each insertion on $\mathrm{C}$ is effectively performed only if the number of steps to be inserted is less than the already stored one.

\subsection{Controller Strengthening Phase}

The second phase of our approach performs a strengthening of the controller $\mathrm{C}$ generated by the first phase. In fact, $\mathrm{C}$ only contains an optimal plan that can be used to drive $\mathcal{S}$ from $s_{0}$ to the goal. That is, $\mathrm{C}$ does not take into account any state outside the optimal plan. The final controller should be aware of a larger set of states: indeed, the dynamics of $\mathcal{S}$ can be very complex, and a particular setting of the control variables may not always drive $\mathcal{S}$ to the expected state. That is, all the state variables usually have a specific tolerance, and the reactions to controls are subject to these tolerances. For this reason, we refer to the controller $\mathrm{C}$ output by the first phase as a raw controller.

Therefore, to ensure the robustness of the controller, in the second strengthening phase we explore a larger number of states obtained by randomly perturbing the raw 
controller states. That is, for each state $s$ in the raw controller table C, we apply a set of small random changes, bounded by the state variables tolerances, and obtain a new state $s^{\prime}$. Then, from each new state $s^{\prime}$, we start a breadth first visit of the state space of $\mathcal{S}$ stopping as soon as we reach a state $s^{\prime \prime}$ that is already in C. The path from $s^{\prime}$ to $s^{\prime \prime}$ is stored in $\mathrm{C}$ and the process is restarted.

After some iterations of this process, we have that $\mathrm{C}$ is now able to drive $\mathcal{S}$ from any reasonable system state to the nearest state of the optimal controller and, from there, reach a goal. That is, $\mathrm{C}$ is now our final optimal controller.

\section{The Controller Generation Process}

The CGMur $\varphi$ tool is an extended version of the CMur $\varphi$ [12] [13] model checker. It is based on an explicit enumeration of the state space, originally developed to verify protocol-like systems. We choose $\mathrm{CMur} \varphi$ as a base to develop our controller generator since it already implements the most common state space compression techniques, such as bit compression [14] and hash compaction [15] [16], useful to decrease the memory requirements of the controller generation process when dealing with large-dimensional control systems. In particular, when bit compression is enabled, CMur $\varphi$ saves memory by using every bit of the state descriptor, the memory structure maintaining the state variables, instead of aligning the state variables on byte boundaries (this saves on average $300 \%$ of memory). When using hash compaction, compressed values, also called state signatures, are used to remember visited states instead of full state descriptors. The compression ratio can be set to obtain an arbitrary state site (CMur $\varphi$ default is 40 bits), but is lossy, so there is a certain probability that some states will have the same signature after compression.

Moreover, the CMur $\varphi$ code is very easy to modify: indeed, in order to generate controllers for complex and hybrid systems we added to CGMur $\varphi$ some important extensions, i.e., finite precision real numbers handling (to model systems with continuous variables) and external linking to $\mathrm{C} / \mathrm{C}++$ functions (to easily model the most complex aspects of the plant, or even interface the model with a complete plant simulator).

The behavioral part of the plant is modeled in CGMur $\varphi$ through a collection of guarded transition rules, whereas the goal construct is used to define the goal properties, that is "normal" or "safe" states of the plant, i.e. the states that the controller should to bring (or maintain) the plant to.

In the following sections we describe the controller generation algorithm that is the core of CGMur $\varphi$. In particular, first we show the data structures used, then we illustrate the procedure for the synthesis and strengthening of the controller table.

\subsection{Data Structures}

The controller generation algorithm of CGMur $\varphi$ uses the following data structures:

- the stack ST contains pairs $(s, r)$, where $s$ is a state and $r$ is the index of last transition (i.e., CMur $\varphi$ rule) fired from $s$. 


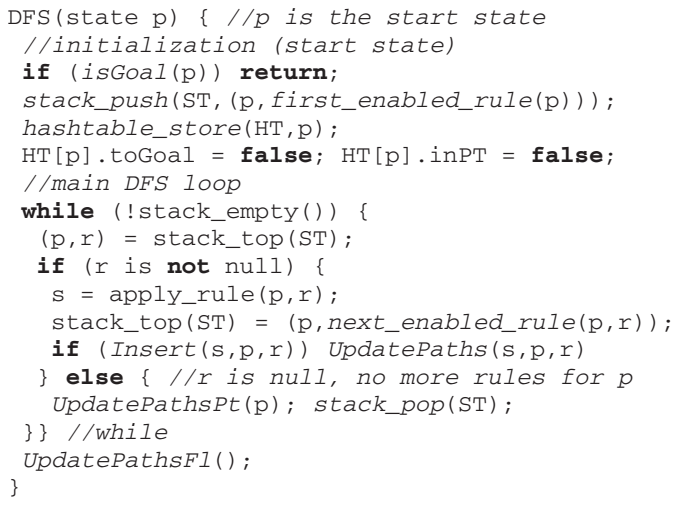

Fig. 1 Extended CGMur $\varphi$ depth first search.

- each slot of the hash table HT contains a (visited) state and two special flags: the toGoal flag indicates that a goal can be reached from this state in one or more steps (transitions), whereas the inPT flag is true if the state has been saved in the predecessors table.

- the predecessors table PT is an hash table storing $(s, l)$ pairs where $s$ is a state on the DFS branch being currently explored, and $l$ is the list of paths leading from an initial state to $s$. Each step of the path contains a state and the action that leads to that state from the previous step.

- the final transitions list FL stores paths to visited states (similarly to the predecessors table) that are discovered when such states are outside the current DFS branch. These paths are merged with the ones in the predecessors table to compute the shortest path to the corresponding states at the end of the state space exploration.

- the controller table CTRL contains, for each reachable system state $s$ that leads (in one or more steps) to a goal, a pair $\langle r, c\rangle$ indicating that the shortest path leading from $s$ to a goal state has $c$ steps, where the first step is the action given by rule $r$.

\subsection{Optimal Raw Controller Synthesis Algorithm}

The optimal raw controller synthesis algorithm, as shown in Fig. 1, consists of an extended depth-first visit of the plant state space. As in a standard DF visit, each state $s$ to be visited is generated by applying a particular rule $r$ to the current state $p$. In Fig. 1, function first_enabled_rule $(p)$ returns the first rule that can be applied on a particular state $p$, whereas function next_enabled_rule $(p, r)$ returns the next rule that can be applied on $\mathrm{p}$ after rule $r$. Both functions return null if such transition does not exist.

In addition, during the DF visit our algorithm updates the controller table when a goal is encountered (in function Insert), when an already visited state is encountered (in function UpdatePaths), when all the children of a state have been explored (in function UpdatePathsPt ( ) ) and when the state space exploration ends (in function UpdatePathsFl).

The function Insert, given a new state $s$ reached from state $p$ by firing rule $r$, checks if $s$ is a goal state and, if so, it creates an entry in the controller table for the state 


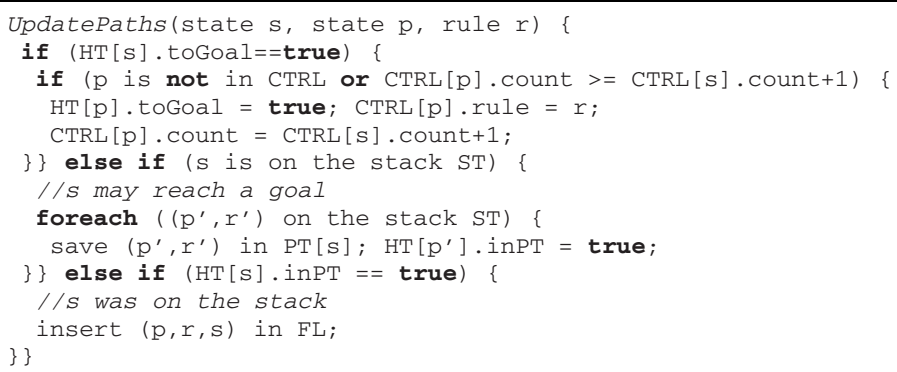

Fig. 2 Function UpdatePaths.

$p$ using the rule $r$. When $s$ is not a goal, Insert behaves as in a standard DFS: if $s$ is non visited the function pushes it on the stack and stores it in the hash table; otherwise, the function simply returns true to indicate that $s$ is a visited state.

Figure 2 shows the details of function UpdatePaths that is called when the DFS reaches an already visited state $s$ by applying a rule $r$ on a state $p$. In this case, we may have to update the controller table CTRL:

- if $s$ reaches a goal, then also $p$ does. Thus, if $p$ is not in the controller table, we insert it together with $r$. Otherwise, if $p$ is already present in the controller table, we update its rule with $r$ if the goal path through $s$ is shorter than the path previously set for $p$ in the controller table. This update ensures the optimality of generated controller.

- if $s$ is in the stack, then it may still reach a goal. Thus we remember all the states on the path leading to $s$ that is represented by the current stack content by saving them in the predecessors table PT.

- finally, if $s$ is in the predecessors table, but not on the stack, we save it in the final list FL, together with its parent $p$ and the transition $r$. This information will be later used to resolve cyclic paths in the predecessor table.

The function UpdatePathsPt is called when a state $s$ has been completely expanded by the DFS algorithm. If $s$ reached a goal, then for each state $p$ in the predecessors table of $s$, we add to CTRL a rule that allows $p$ to reach the goal through $s$.

Finally, the function UpdatePathsF1, called at the end of the visit, completes the controller table by adding rules for states in the final list FL. This is similar to what is done by UpdatePathsPt, but is applied at the end of the state space exploration and on a separate set of states. Such states belong to intersecting paths of the transition graph, so their shortest path to the goal can be computed only when all the goal paths have been generated.

\subsection{Controller Strengthening Algorithm}

The controller strengthening is implemented by the exploreNeighborhood function shown in Fig. 3. For each state $p$ in the controller table, the function generates MAX_VARS_PER_STATE variations by applying small changes to the state variables. Then, the algorithm checks if each of the new states is in turn in the controller table. If 


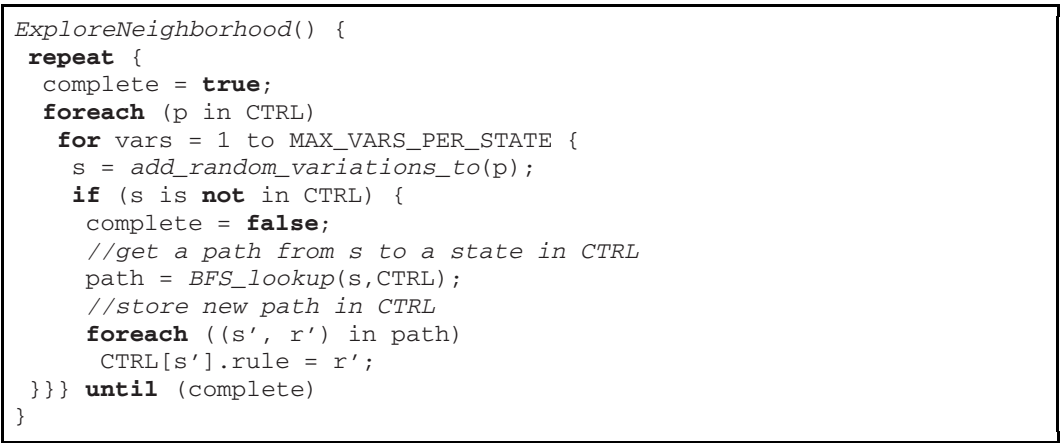

Fig. 3 Function exploreNeighborhood.

any generated state $s$ is not yet handled by the controller, the function performs a BFS search from $s$ until it reaches a controlled state, and inserts the path from $s$ to such state in CTRL. The process is repeated until all the generated variations are found in CTRL. At this point, CTRL knows how to drive the plant on the optimal plan and how to bring the plant on the nearest optimal plan state from a reasonable number of states outside the optimal plan.

\section{Truck-and-Trailer Obstacles Avoiding Controller}

To show the effectiveness of our approach, we show how it can be applied to the truck and trailer with obstacles avoidance problem.

The goal of a truck and trailer controller is to back a truck with a trailer up to a parking place starting from any initial position in the parking lot. This is a non trivial problem due to the dynamics of the truck-trailer pair (see the mathematical model in Sect. 5.1).

Moreover, we added to the parking lot some obstacles, which have to be avoided by the truck while maneuvering to reach the parking place. In this setting, also finding a suitable maneuver to reach the goal for any starting position may be an hard task. On the other hand, finding an optimal maneuver is a very complex problem, that cannot be modeled and resolved using common mathematical or programming strategies, e.g., using a dynamic programming approach.

Indeed, in the truck-and-trailer-with-obstacles problem, a backward decomposition of the cost function (e.g., the length of the path) is hard to perform, due to the complexity of the system dynamics function and to the presence of obstacles, whereas a forward decomposition does not satisfy the optimality principle, since the presence of obstacles may make an optimal local maneuver not optimal w.r.t. the final goal. This also makes fuzzy controllers not suitable for this problem, since fuzzy rules have a local character.

In the following sections we give details of the truck and trailer model and show the results obtained by applying the controller generation process described in Sect. 3 to perform a systematic analysis of the truck trajectories, discretized as a sequence of forward steps. 


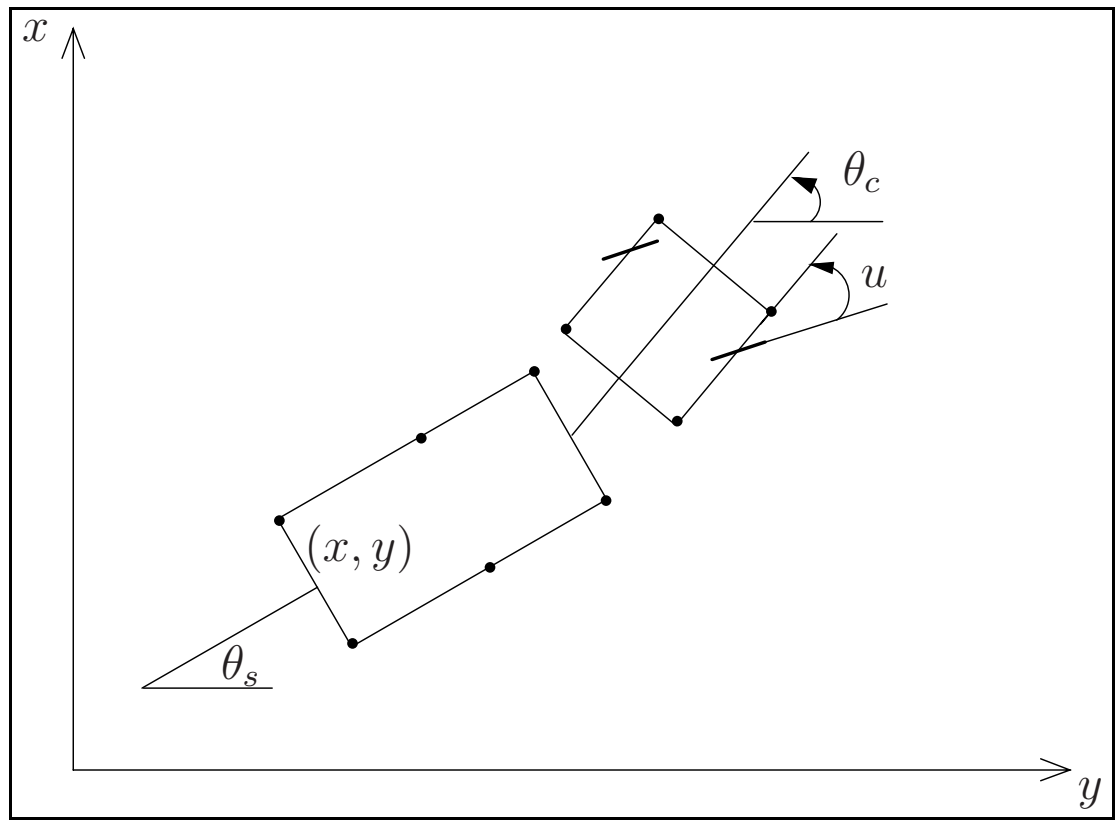

Fig. 4 Truck and trailer system description.

\subsection{Model Description}

Our model of the truck and trailer is based on the set of equations presented in [17]. The system has four state variables, that is the coordinates of the center rear of the trailer $(x, y \in[0,50])$, the angle of the trailer w.r.t. the $x$-axis $\left(\theta_{S} \in\left[-90^{\circ}, 270^{\circ}\right]\right)$ and the angle of the cab w.r.t the $x$-axis $\left(\theta_{C} \in\left[-90^{\circ}, 270^{\circ}\right]\right)$. We assume that the truck moves backward with constant speed of $2 \mathrm{~m} / \mathrm{s}$, so the only control variable is the steering angle $u \in\left[-70^{\circ}, 70^{\circ}\right]$. Figure 4 shows a schematic view of the truck and trailer system with its state and control variable. Moreover we single out 10 points in the truck and trailer border (displayed in the Fig. 4 by bold points) representative of the truck and trailer position.

If the values of the state variables at time $t$ are $x[t], y[t], \theta_{S}[t]$ and $\theta_{C}[t]$, and the steering angle is $u$, then the new values of state variables at time $t+1$ are determined by following equations:

$$
\begin{gathered}
x[t+1]=x[t]-B * \cos \left(\theta_{S}[t]\right) \\
y[t+1]=y[t]-B * \sin \left(\theta_{S}[t]\right) \\
\theta_{S}[t+1]=\theta_{S}[t]-\arcsin \left(\frac{A * \sin \left(\theta_{C}[t]-\theta_{S}[t]\right)}{L_{S}}\right) \\
\theta_{C}[t+1]=\theta_{C}[t]+\arcsin \left(\frac{r * \sin (u)}{L_{S}+L_{C}}\right)
\end{gathered}
$$


where $A=r * \cos (u), B=A * \cos \left(\theta_{C}[t]-\theta_{S}[t]\right), r=1$ is the truck movement length per time step, $L_{S}=4$ and $L_{C}=2$ are the length of the trailer and cab, respectively (all the measures are in meters).

After computing (3) and (4), the new value of $\theta_{C}$ is adjusted to respect the jackknife constraint: $\left|\theta_{S}-\theta_{C}\right| \leq 90^{\circ}$.

Note that this model does not consider the obstacles: indeed, embedding the obstacle avoidance in the mathematical description of the truck and trailer dynamics would result in a untractable set of equations. This feature will be added directly in the CGMur $\varphi$ model described below.

\subsection{The CGMur $\varphi$ Model}

In the CGMur $\varphi$ model we use real values to represent the state variables $x$ and $y$, whilst for the angle values (i.e., $\theta_{S}, \theta_{C}$ and $u$ ) it is sufficient, w.r.t. the system dimensions, to use integer values. Moreover, we define some tolerance constants to set up a range of admissible final positions and angles for the center rear of the trailer. These tolerances are used to define the CGMur $\varphi$ goal property.

To embed the obstacles in the model, we approximate them through their bounding rectangles (or rectangle compositions). Then we consider the representative points of the truck-trailer position (defined above, see Sect. 5.1) and, each time a new truck position is computed, we use a function to check if any of these points has hit the parking lot obstacles or borders. Therefore, our controller synthesis algorithm considers only feasible maneuvers to the goal state.

Moreover, in order to obtain a more robust controller we also considered the maneuvering errors due to the truck-trailer complex dynamic properties (e.g., friction, brakes response time, etc.) that cannot be easily embedded in the mathematic model. We used such errors to draw a security border around each obstacle and used these augmented obstacles in the collision check described above.

To estimate maximum maneuvering error we applied a Monte Carlo's method described as follows. We consider a large set of valid parking lot positions $S=\left\{s_{k} \mid 1 \leq\right.$ $k \leq 500000\}$. Given a position $s_{k} \in S$, (1) we apply a random maneuver $m_{k}$ obtaining the new position $\bar{s}_{k}$. Then (2) we randomly perturb $s_{k}$ generating the position $s_{k}^{p}$ and apply the same maneuver $m_{k}$ on $s_{k}^{p}$ obtaining the position $\bar{s}_{k}^{p}$. Finally, (3) we compute the distance of the selected truck points $P_{i}$ between the positions $s_{k}^{p}$ and $\bar{s}_{k}^{p}$. This process is repeated 200 times for each position in $S$, thus analyzing 100 millions of perturbations. The security border size is the highest distance measured for a point in the step (3). We found out that this distance is $0.98 \mathrm{~m}$.

\subsection{Experimental Results}

We tested our methodology using several obstacles topologies. In this section we present the results relative to the map shown in Fig. 5 where the obstacles and the security borders are highlighted.

Table 1 shows the results of the first phase of our algorithm (see Sect. 4.2). We repeated the controller generation using two different approximations for the real state variables $x$ and $y$, rounding them to 0.5 and 0.2 meters. 


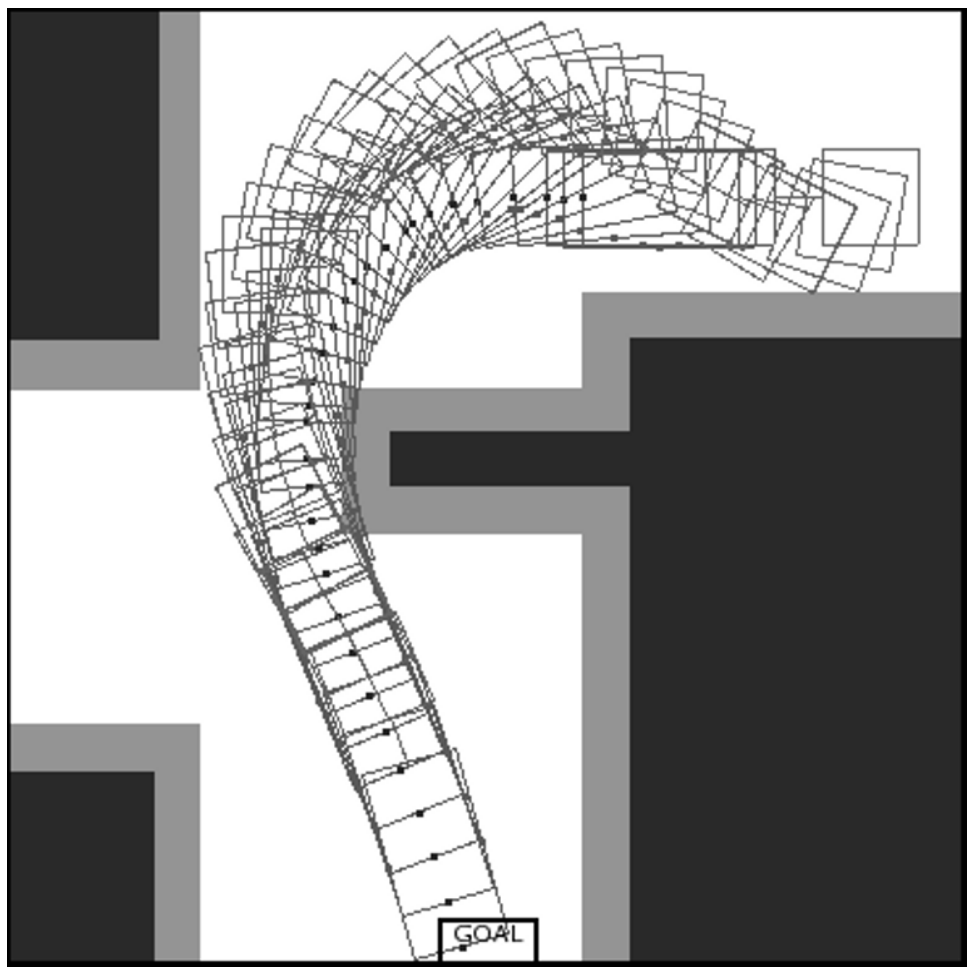

Fig. 5 Optimal trajectory generated by CGMur $\varphi$ from initial position $x=12, y=16, \theta_{s}=0$, $\theta_{c}=0$.

Indeed, an higher precision extends the reachable state space and, consequently, the number of transitions in the controller. The results in Table 1 show that we are able to deal with system having millions of states.

In the second phase, we refined the controller by applying 36 disturbs to each state in the controller table and finding the appropriate rules to reconnect each new state to the optimal controller paths, as described in Sect. 4.3. As shown in Table 2, in this phase a significant number of transitions is added, due to the complexity of the truck-trailer dynamics.

Table 1 Experimental results for optimal raw controller synthesis

\begin{tabular}{|c|c|c|c|c|}
\hline Round & $\begin{array}{c}\text { Reachable } \\
\text { states }\end{array}$ & $\begin{array}{c}\text { Rules } \\
\text { fired }\end{array}$ & $\begin{array}{c}\text { Trans. in } \\
\text { controller }\end{array}$ & $\begin{array}{c}\text { Time } \\
\text { Sec }\end{array}$ \\
\hline $0.5 \mathrm{~m}$ & 2233997 & 64785913 & 382262 & 8160 \\
\hline $0.2 \mathrm{~m}$ & 12227989 & 354611681 & 1749586 & 32847 \\
\hline
\end{tabular}


Table 2 Experimental results for controller strengthening

\begin{tabular}{|c|c|c|c|c|}
\hline Round & $\begin{array}{c}\text { MAX } \\
\text { VARS }\end{array}$ & $\begin{array}{c}\text { Trans. } \\
\text { added }\end{array}$ & $\begin{array}{c}\text { Trans. in } \\
\text { controller }\end{array}$ & $\begin{array}{c}\text { Size of } \\
\text { controller }\end{array}$ \\
\hline $0.5 \mathrm{~m}$ & 36 & 257850 & 640112 & $14 \mathrm{Mb}$ \\
\hline $0.2 \mathrm{~m}$ & 36 & 646364 & 2395950 & $50 \mathrm{Mb}$ \\
\hline
\end{tabular}

Controller Robustness. In order to check the controller robustness, we considered all the trajectories starting from each state in the controller. For each trajectory state $s$, we applied a random disturbance on the state variables, generating a possibly new state $s^{p}$, and then we applied to $s^{p}$ the rule associated to controller state $s^{\prime}$ that is nearest to $s^{p}$. A trajectory is robust if, applying the disturbances above, it eventually reaches the goal state.

Table 3 Check of controller robustness

\begin{tabular}{|c|c|c|c|}
\hline Round & $\begin{array}{c}\text { Disturb range } \\
\text { for } x, y\end{array}$ & $\begin{array}{c}\text { Disturb range } \\
\text { for } \theta_{s}, \theta_{c}\end{array}$ & $\begin{array}{c}\text { Robust } \\
\text { trajectories }\end{array}$ \\
\hline $0.5 \mathrm{~m}$ & $\pm 0.25 \mathrm{~m}$ & $\pm 1^{\circ}$ & $40 \%$ \\
& $\pm 0.125 \mathrm{~m}$ & $\pm 0.5^{\circ}$ & $45 \%$ \\
& $\pm 0.0625 \mathrm{~m}$ & $\pm 0.25^{\circ}$ & $57 \%$ \\
\hline $0.2 \mathrm{~m}$ & $\pm 0.1 \mathrm{~m}$ & $\pm 1^{\circ}$ & $74 \%$ \\
& $\pm 0.05 \mathrm{~m}$ & $\pm 0.5^{\circ}$ & $88 \%$ \\
\hline
\end{tabular}

We checked all trajectories by applying different disturb ranges. As shown in Table 3 , the fraction of robust trajectories increases with the controller precision (i.e., the real values approximation). Note that the percentages of robust trajectories in the second round are completely satisfying considering:

- the optimality of the trajectories;

- the extreme complexity of this parking problem;

- the unavailability of correction maneuvers.

\section{Conclusions}

The controller tables generated through our methodology contain millions of state-rule pairs. Thus, if we are working with small embedded systems, the table size could be a potential issue. This problem can be mitigated by applying various compression techniques on the table.

A completely different solution that we are also experimenting is the generation of hybrid controllers, that are optimal controllers working in parallel with e.g. a fuzzy controller. In this case, the optimal controller ensures the execution of the optimal plans (i.e., it is the optimal raw controller generated in Sect. 4.2), whereas the fuzzy controller is able to bring the system back to the optimal plans from any state outside the optimal 
raw controller. Thus, the fuzzy controller substitutes the extended knowledge generated by the algorithm in Sect. 4.3 with a set of inference rules. These rules may be in turn generated by an iterative learning process driven by an algorithm similar to the one of Sect. 4.3.

\section{References}

1. Kautz, H., Thomas, W., Vardi, M.Y.: 05241 executive summary - synthesis and planning. In Kautz, H., Thomas, W., Vardi, M.Y., eds.: Synthesis and Planning. Number 05241 in Dagstuhl Seminar Proceedings (2006)

2. Lygeros, J., Tomlin, C., Sastry, S.: Controllers for reachability specifications for hybrid systems Special Issue on Hybrid Systems, 35 (1999)

3. Åstrom, K.J., Hägglund, T.: PID controllers - Theory, Design, and Tuning. International Society for Measurement and Con; 2nd edn (2005)

4. Li, H., Gupta, M.: Fuzzy Logic and Intelligent Systems. Kluwer Academic Publishers (1995)

5. Jin, J.: Advanced Fuzzy Systems Design and Applications. Physica-Verlag (2003)

6. Bertsekas, D.P.: Dynamic Programming and Optimal Control. Athena Scientific (2005)

7. Sniedovich, M.: Dynamic Programming. Marcel Dekker (1992)

8. Burch, J.R., Clarke, E.M., McMillan, K.L., Dill, D.L., Hwang, L.J.: Symbolic model checking: $10^{20}$ states and beyond. Information and Computation 98 (1992) 142-170

9. Holzmann, G.J.: The SPIN Model Checker. Addison-Wesley (2003)

10. Dill, D.L., Drexler, A.J., Hu, A.J., Yang, C.H.: Protocol verification as a hardware design aid. In: Proceedings of the 1991 IEEE International Conference on Computer Design on VLSI in Computer \& Processors, IEEE Computer Society (1992) 522-525

11. Hu, A.J., York, G., Dill, D.L.: New techniques for efficient verification with implicitly conjoined bdds. In: DAC '94: Proceedings of the 31st Annual Conference on Design Automation, New York, USA, ACM Press (1994) 276-282

12. http://www.dsi.uniroma1.it/ tronci/cached.murphi.html (2006)

13. Della Penna, G., Intrigila, B., Melatti, I., Tronci, E., Venturini Zilli, M.: Exploiting transition locality in automatic verification of finite state concurrent systems. STTT 6 (2004) 320-341

14. Murphi Web Page: http://sprout.stanford.edu/dill/murphi.html (2004)

15. Stern, U., Dill, D.: Using magnetic disk instead of main memory in the mur $\varphi$ verifier. In $\mathrm{Hu}$, A.J., Vardi, M.Y., eds.: Computer Aided Verification, 10th International Conference, CAV '98, Vancouver, BC, Canada, June 28-July 2, Proceedings. Volume 1427 of Lecture Notes in Computer Science, Springer (1998) 172-183

16. Stern, U., Dill, D.L.: Improved probabilistic verification by hash compaction. In: CHARME '95: Proceedings of the IFIP WG 10.5 Advanced Research Working Conference on Correct Hardware Design and Verification Methods, London, UK, Springer-Verlag (1995) 206-224

17. Nguyen, D., Widrow, B.: The truck backer-upper: an example of self learning in neural networks. In: W.T. Miller, R.S. Sutton, and P.J. Werbos, eds.: Neural Networks For Control, Mit Press Series In Neural Network Modeling and Connectionism. MIT Press, Cambridge, MA (1990) 287-299 